\author{
B.R. Bayraktar ${ }^{1}$, A.Kh. Attaev, ${ }^{2}$ \\ ${ }^{1}$ Uludă University, Bursa, Turkey; \\ ${ }^{2}$ IAMA KBSC RAS, Nalchik, Russia \\ (E-mail: bbayraktar@uludag.edu.tr, attaev.anatoly@yandex.ru)
}

\title{
Fractional Integral Inequalities for Some Convex Functions
}

\begin{abstract}
In this paper, we obtained several new integral inequalities using fractional Riemann-Liouville integrals for convex s-Godunova-Levin functions in the second sense and for quasi-convex functions. The results were gained by applying the double Hermite-Hadamard inequality, the classical Holder inequalities, the power mean, and weighted Holder inequalities. In particular, the application of the results for several special computing facilities was given. Some applications to special means for arbitrary real numbers: arithmetic mean, logarithmic mean, and generalized log-mean, are provided.
\end{abstract}

Keywords: integral inequality, Hermite-Hadamard inequalitiy, convex function, s-Godunova-Levin convex, quasiconvex, fractional integral, Hölder inequality, power mean inequality.

\section{Introduction}

Here, we give some well-known definitions in the literature that have attracted the attention of many scientists in the field of convex analysis.

Definition 1. A function $\psi$ defined on the interval $\left[v_{1}, v_{2}\right] \subset \mathbb{R}$ is convex on this interval if the inequality

$$
\psi(\tau \xi+(1-\tau) \zeta) \leq \tau \psi(\xi)+(1-\tau) \psi(\zeta)
$$

holds for all $\xi, \zeta \in\left[v_{1}, v_{2}\right]$ and $\tau \in[0,1]$.

Definition 2. ([1]) We say that $\psi: I \subset \mathbb{R} \rightarrow \mathbb{R}$ is a Godunova-Levin function or that $\psi$ belongs to the class $Q(I)$, if $f$ is non-negative and

$$
\psi(\tau \xi+(1-\tau) \zeta) \leq \frac{\psi(\xi)}{\tau}+\frac{\psi(\zeta)}{1-\tau}, \forall \xi, \zeta \in I, \tau \in(0,1)
$$

Definition 3. ([2]) A function $\psi:\left[v_{1}, v_{2}\right] \rightarrow \mathbb{R}$ is said to be $s$-Godunova-Levin convex, with $s \in[0,1]$, if inequality

$$
\psi(\tau \xi+(1-\tau) \zeta) \leq \frac{\psi(\xi)}{\tau^{s}}+\frac{\psi(\zeta)}{(1-\tau)^{s}}
$$

holds for all $\tau \in(0,1)$ and $\xi, \zeta \in\left[v_{1}, v_{2}\right]$.

Definition 4. ([2]) A function $\psi: I \subseteq \mathbb{R} \rightarrow \mathbb{R}$ is $P$ function or that $f$ belongs to the class of $P(I)$; if it is non-negative and for all $\xi, \zeta \in I$ and $\tau \in[0 ; 1]$; satisfies the following inequality:

$$
\psi(\tau \xi+(1-\tau) \zeta) \leq \psi(\xi)+\psi(\zeta)
$$

It is obvious that $s$-Godunova-Levin type functions for $s=0$ yield $P$ functions.

Definition 5. ([3]) A function $\psi$ defined on the interval $\left[v_{1}, v_{2}\right]$, if it satisfies the inequality

$$
\psi(\tau \xi+(1-\tau) \zeta) \leq \max \{\psi(\xi), \psi(\zeta)\}
$$

for all $\xi, \zeta \in I \subset \mathbb{R}$ and $\tau \in[0,1]$, then the function is called quasi-convex on this interval or $\psi$ belongs to the class $Q C(I)$.

It was established that any convex function is a quasiconvex function, but the converse is not true.

\footnotetext{
* Corresponding author.

E-mail: attaev.anatoly@yandex.ru
} 
There are many classes of convexity of functions in the literature. These functional classes, which have different properties, often generalize each other. Over the past few decades, researchers have obtained a few interesting results related to mathematical inequalities using fractional integration operators for various classes of convex functions.

Fractional integral operators generalize the notion of definite integration and have a significant effect on generalizing mathematical inequalities, so these operators play a vital role in the development of science and technology. In recent decades, many different fractional integration operators have been formulated, many of which supplement and extend the classical operators. A special place among these operators is occupied by the Riemann - Liouville fractional integration operators.

In recent years, scientists have obtained some mathematical inequalities associated with various operators, for example, see [4-7]. Among these operators, the Riemann-Liouville operators, which have become classical, occupy a special place.

Definition 6. ([8]) Let $\psi \in L\left[v_{1}, v_{2}\right]$. Then the left and right Riemann-Liouville integrals of order $\alpha>0$ with $v_{1} \geq 0$ are defined by

$$
J_{v_{1}^{+}}^{\alpha} \psi(\xi)=\frac{1}{\Gamma(\alpha)} \int_{v_{1}}^{\xi}(\xi-\tau)^{\alpha-1} \psi(\tau) d \tau, \quad \xi>v_{1}
$$

and

$$
J_{v_{2}-}^{\alpha} \psi(\xi)=\frac{1}{\Gamma(\alpha)} \int_{\xi}^{v_{2}}(\tau-\xi)^{\alpha-1} \psi(\tau) d \tau, \quad \xi<v_{2}
$$

respectively, where $\Gamma(\alpha)=\int_{0}^{\infty} e^{-u} u^{\alpha-1} d u$. Here $J_{v_{1}^{+}}^{0} \psi(\xi)=J_{v_{2}^{-}}^{0} \psi(\xi)=\psi(\xi)$. In the case of $\alpha=1$, the fractional integral reduces to the classical integral.

In the theory of inequalities, a important role is played by the double Hermite-Hadamard inequality:

Let $\psi: I \subseteq \mathbb{R} \rightarrow \mathbb{R}$ be a convex function and $v_{1}, v_{2} \in I$, with $v_{1}<v_{2}$. The following double inequality holds

$$
\psi\left(\frac{v_{1}+v_{2}}{2}\right) \leq \frac{1}{v_{2}-v_{1}} \int_{v_{1}}^{v_{2}} \psi(\xi) d \xi \leq \frac{\psi\left(v_{1}\right)+\psi\left(v_{2}\right)}{2} .
$$

If $\psi$ is a concave function, then the inequalities are in the opposite direction.

A few studies (for example, [5-16] and references therein) are devoted to obtaining, improving, and generalizing integral inequalities in terms of various fractional integral operators.

It is known that the class of quasiconvex functions includes the class of convex functions defined on finite closed intervals. Some references to quasiconvex functions and their applications can be seen in [7], [17-21] and references therein. Studies devoted to Godunova-Levin type convex functions can be seen, for example, in the works [22-27] and references therein.

In addition to the classical integral Hölder inequality and its version-the power mean, we use the weighted Hölder inequality $([28])$ :

$$
\left|\int_{I} \psi(\tau) s(\tau) h(\tau) d \tau\right| \leq\left(\int_{I}|\psi(\tau)|^{p} h(\tau) d \tau\right)^{\frac{1}{p}}\left(\int_{I}|s(\tau)|^{q} h(\tau) d \tau\right)^{\frac{1}{q}}
$$

for $p>1, \frac{1}{p}+\frac{1}{q}=1$ and $h(\tau) \geq 0, \forall \tau \in I$.

Bayraktar in [9], proved the following identity:

Lemma 1. (Lemma 2.1, for $m=1$ ) Let $\psi: I \subset \mathbb{R} \rightarrow \mathbb{R}$ be a twice differentiable function on $I^{\circ}$, where $v_{1}, v_{2} \in I$ with $\tau \in[0,1]$. If $\psi^{\prime \prime} \in L\left[v_{1}, v_{2}\right]$. Then $\forall \alpha>1$, with properties of Gamma function we have

$$
\begin{gathered}
\frac{2^{\alpha-2} \Gamma(\alpha)}{\left(v_{2}-v_{1}\right)^{\alpha-1}}\left[J_{\frac{v_{1}+v_{2}}{2}}^{\alpha-1}+\psi\left(v_{2}\right)+J_{\frac{v_{1}+v_{2}}{2}}^{\alpha-1}-\psi\left(v_{1}\right)\right]-\psi\left(\frac{v_{1}+v_{2}}{2}\right) \\
=\frac{\left(v_{2}-v_{1}\right)^{2}}{\alpha 2^{2-\alpha}}\left[\int_{0}^{\frac{1}{2}} \tau^{\alpha} \psi^{\prime \prime}\left(\tau v_{1}+(1-\tau) v_{2}\right) d \tau\right. \\
\left.+\int_{\frac{1}{2}}^{1}(1-\tau)^{\alpha} \psi^{\prime \prime}\left(\tau v_{1}+(1-\tau) v_{2}\right) d \tau\right] .
\end{gathered}
$$


In [15], Sarıkaya et al. proved the following identity:

Lemma 2. ([15]) Let $\psi:\left[v_{1}, v_{2}\right] \rightarrow R$, be a differentiable mapping on $\left(v_{1}, v_{2}\right)$. If $\psi^{\prime} \in L\left[v_{1}, v_{2}\right]$, then the equality:

$$
\begin{gathered}
\frac{\psi\left(v_{1}\right)+\psi\left(v_{2}\right)}{2}-\frac{\Gamma(\alpha+1)}{2\left(v_{2}-v_{1}\right)^{\alpha}}\left[J_{v_{1}^{+}}^{\alpha} \psi\left(v_{2}\right)+J_{v_{2}^{-}}^{\alpha} \psi\left(v_{1}\right)\right]= \\
=\frac{v_{2}-v_{1}}{2} \int_{0}^{1}\left[(1-\tau)^{\alpha}-\tau^{\alpha}\right] \psi^{\prime}\left(\tau v_{1}+(1-\tau) v_{2}\right) d \tau
\end{gathered}
$$

holds, where $\alpha>0$.

The main goal of the article is to obtain new integral inequalities in terms of fractional integration operators of the Riemann-Liouville type on the basis of the formulated identities for $s$-Godunova-Levin and quasiconvex functions.

\section{Main results}

Theorem 1. Let $\psi: I=\left[v_{1}, v_{2}\right] \rightarrow \mathbb{R}$ be a differentiable function on $\left(v_{1}, v_{2}\right)$ such that $\psi^{\prime \prime} \in L\left[v_{1}, v_{2}\right]$. If $\left|\psi^{\prime \prime}\right|^{q} \in Q C(I)$ and $q \geq 1$, then the inequality

$$
\begin{aligned}
& \left|\frac{2^{\alpha-2} \Gamma(\alpha)}{\left(v_{2}-v_{1}\right)^{\alpha-1}}\left[J_{\frac{v_{1}+v_{2}}{2}}^{\alpha-1} \psi\left(v_{2}\right)+J_{\frac{v_{1}+v_{2}}{2}}^{\alpha-1} \psi\left(v_{1}\right)\right]-\psi\left(\frac{v_{1}+v_{2}}{2}\right)\right| \leq \\
& \leq\left(\frac{v_{2}-v_{1}}{2}\right)^{2} \frac{1}{\alpha(\alpha+1)}\left[\max \left(\left|\psi^{\prime \prime}\left(v_{1}\right)\right|^{q},\left|\psi^{\prime \prime}\left(v_{2}\right)\right|^{q}\right)\right]^{\frac{1}{q}}
\end{aligned}
$$

holds, where $\alpha>1$.

Proof. From (1) using the properties of the modulus and the power mean inequality, we get

$$
\begin{aligned}
& \frac{2^{\alpha-2} \Gamma(\alpha)}{\alpha\left(v_{2}-v_{1}\right)^{\alpha-1}}\left[J_{\frac{v_{1}+v_{2}}{2}}^{\alpha-1} \psi\left(v_{2}\right)+J_{\frac{v_{1}+v_{2}}{2}}^{\alpha-1}-\psi\left(v_{1}\right)\right]-\psi\left(\frac{v_{1}+v_{2}}{2}\right)=U \\
|U| \leq & \frac{\left(v_{2}-v_{1}\right)^{2}}{\alpha 2^{2-\alpha}}\left[\int_{0}^{\frac{1}{2}} \tau^{\alpha}\left|\psi^{\prime \prime}\left(\tau v_{1}+(1-\tau) v_{2}\right)\right| d \tau+\right. \\
& \left.+\int_{\frac{1}{2}}^{1}(1-\tau)^{\alpha}\left|\psi^{\prime \prime}\left(\tau v_{1}+(1-\tau) v_{2}\right)\right| d \tau\right] \leq \\
& \leq \frac{\left(v_{2}-v_{1}\right)^{2}}{\alpha 2^{2-\alpha}}\left[\left(\int_{0}^{\frac{1}{2}} \tau^{\alpha} d \tau\right)^{1-\frac{1}{q}}\left(\int_{0}^{\frac{1}{2}} \tau^{\alpha}\left|\psi^{\prime \prime}\left(\tau v_{1}+(1-\tau) v_{2}\right)\right|^{q} d \tau\right)^{\frac{1}{q}}+\right. \\
& \left.+\left(\int_{\frac{1}{2}}^{1}(1-\tau)^{\alpha} d \tau\right)^{1-\frac{1}{q}}\left(\int_{\frac{1}{2}}^{1}(1-\tau)^{\alpha}\left|\psi^{\prime \prime}\left(\tau v_{1}+(1-\tau) v_{2}\right)\right|^{q} d \tau\right)^{\frac{1}{q}}\right]= \\
& =\frac{\left(v_{2}-v_{1}\right)^{2}}{\alpha 2^{2-\alpha}} \frac{1}{2^{\alpha}(\alpha+1)}\left[\max \left(\left|\psi^{\prime \prime}\left(v_{1}\right)\right|^{q},\left|\psi^{\prime \prime}\left(v_{2}\right)\right|^{q}\right)\right]^{\frac{1}{q}}= \\
& =\left(\frac{v_{2}-v_{1}}{2}\right)^{2} \frac{1}{\alpha(\alpha+1)}\left[\max \left(\left|\psi^{\prime \prime}\left(v_{1}\right)\right|^{q},\left|\psi^{\prime \prime}\left(v_{2}\right)\right|^{q}\right)\right]^{\frac{1}{q}}
\end{aligned}
$$

Here, we used the quasi-convex of $\left|\psi^{\prime \prime}\right|^{q}$ on $\left[v_{1}, v_{2}\right]$ and it can be easily checked that

$$
\int_{\frac{1}{2}}^{1}(1-\tau)^{\alpha} d \tau=\int_{0}^{\frac{1}{2}} \tau^{\alpha} d \tau=\frac{1}{2^{\alpha+1}(\alpha+1)} .
$$

This completes the proof. 
Theorem 2. Let $\psi: I=\left[v_{1}, v_{2}\right] \rightarrow \mathbb{R}$ be a differentiable function on $\left(v_{1}, v_{2}\right)$ such that $\psi^{\prime \prime} \in L\left[v_{1}, v_{2}\right]$. If $\left|\psi^{\prime \prime}\right|$ is $s$-Godunova-Levin function on $\left[v_{1}, v_{2}\right]$ and $q \geq 1$, then the inequality

$$
\begin{aligned}
& \left|\frac{2^{\alpha-2} \Gamma(\alpha)}{\left(v_{2}-v_{1}\right)^{\alpha-1}}\left[J_{\frac{v_{1}+v_{2}}{2}}^{\alpha-1} \psi\left(v_{2}\right)+J_{\frac{v_{1}+v_{2}}{2}}^{\alpha-1}-\psi\left(v_{1}\right)\right]-\psi\left(\frac{v_{1}+v_{2}}{2}\right)\right| \\
& \leq \frac{\left(v_{2}-v_{1}\right)^{2}\left[\left|\psi^{\prime \prime}\left(v_{1}\right)\right|+\left|\psi^{\prime \prime}\left(v_{2}\right)\right|\right]}{2^{2-\alpha}}\left[B_{\frac{1}{2}}(\alpha+1, \alpha-s+1)+\frac{1}{2^{\alpha-s+1}(\alpha-s+1)}\right]
\end{aligned}
$$

holds, where $\alpha>1, s \in[0,1], B_{x}(a, b)=\int_{0}^{x} \tau^{a-1}(1-\tau)^{b-1} d \tau$ is the incomplete Euler Beta function.

Proof. From (1) using the properties of the modulus and the power mean inequality, we get

$$
\begin{aligned}
\frac{2^{\alpha-2} \Gamma(\alpha)}{\left(v_{2}-v_{1}\right)^{\alpha-1}} & {\left[J_{\frac{v_{1}+v_{2}}{2}}^{\alpha-1}+\left(v_{2}\right)+J_{\frac{v_{1}+v_{2}}{2}}^{\alpha-1} \psi\left(v_{1}\right)\right]-\psi\left(\frac{v_{1}+v_{2}}{2}\right)=U } \\
|U| \leq & \frac{\left(v_{2}-v_{1}\right)^{2}}{2^{2-\alpha}}\left[\int_{0}^{\frac{1}{2}} \tau^{\alpha}\left|\psi^{\prime \prime}\left(\tau v_{1}+(1-\tau) v_{2}\right)\right| d \tau\right. \\
& \left.+\int_{\frac{1}{2}}^{1}(1-\tau)^{\alpha}\left|\psi^{\prime \prime}\left(\tau v_{1}+(1-\tau) v_{2}\right)\right| d \tau\right] \\
& \leq \frac{\left(v_{2}-v_{1}\right)^{2}}{\alpha 2^{2-\alpha}}\left[\int_{0}^{\frac{1}{2}} \tau^{\alpha}\left|\psi^{\prime \prime}\left(\tau v_{1}+(1-\tau) v_{2}\right)\right| d \tau\right. \\
& \left.+\int_{\frac{1}{2}}^{1}(1-\tau)^{\alpha}\left|\psi^{\prime \prime}\left(\tau v_{1}+(1-\tau) v_{2}\right)\right| d \tau\right]
\end{aligned}
$$

Sinse $\left|\psi^{\prime \prime}\right|$ is a $s$-Godunova-Levin type function on $\left[v_{1}, v_{2}\right]$ for the integrals we can write

$$
\begin{aligned}
& \int_{0}^{\frac{1}{2}} \tau^{\alpha}\left|\psi^{\prime \prime}\left(\tau v_{1}+(1-\tau) v_{2}\right)\right| d \tau \leq \\
\leq & \left|\psi^{\prime \prime}\left(v_{1}\right)\right| \int_{0}^{\frac{1}{2}} \tau^{\alpha-s} d \tau+\left|\psi^{\prime \prime}\left(v_{2}\right)\right| \int_{0}^{\frac{1}{2}} \tau^{\alpha}(1-\tau)^{\alpha-s} d \tau \leq \\
\leq & \frac{\left|\psi^{\prime \prime}\left(v_{1}\right)\right|}{2^{\alpha-s+1}(\alpha-s+1)}+B_{\frac{1}{2}}(\alpha+1, \alpha-s+1)\left|\psi^{\prime \prime}\left(v_{2}\right)\right| .
\end{aligned}
$$

And for the integral, we get

$$
\begin{aligned}
& \int_{\frac{1}{2}}^{1}(1-\tau)^{\alpha}\left|\psi^{\prime \prime}\left(\tau v_{1}+(1-\tau) v_{2}\right)\right|^{q} d \tau \leq \\
\leq & \left|\psi^{\prime \prime}\left(v_{1}\right)\right| \int_{\frac{1}{2}}^{1} \tau^{\alpha-s}(1-\tau)^{\alpha} d \tau+\left|\psi^{\prime \prime}\left(v_{2}\right)\right| \int_{\frac{1}{2}}^{1}(1-\tau)^{\alpha-s} d \tau \leq \\
\leq & B_{\frac{1}{2}}(\alpha+1, \alpha-s+1)\left|\psi^{\prime \prime}\left(v_{1}\right)\right|+\frac{\left|\psi^{\prime \prime}\left(v_{2}\right)\right|}{2^{\alpha-s+1}(\alpha-s+1)} .
\end{aligned}
$$

In this way

$$
\begin{aligned}
|U| \leq & \frac{\left(v_{2}-v_{1}\right)^{2}}{2^{2-\alpha}}\left(B_{\frac{1}{2}}(\alpha+1, \alpha-s+1)+\frac{1}{2^{\alpha-s+1}(\alpha-s+1)}\right) \times \\
& \times\left(\left|\psi^{\prime \prime}\left(v_{1}\right)\right|+\left|\psi^{\prime \prime}\left(v_{2}\right)\right|\right) .
\end{aligned}
$$

This completes the proof.

Corollary 1. If we choose $s=1$ and $\alpha=2$ in Theorem, then for Godunova-Levin function, we get the inequality

$$
\left|\frac{1}{v_{2}-v_{1}} \int_{v_{1}}^{v_{2}} \psi(\tau) d \tau-\psi\left(\frac{v_{1}+v_{2}}{2}\right)\right| \leq \frac{29\left(v_{2}-v_{1}\right)^{2}}{192}\left[\left|\psi^{\prime \prime}\left(v_{1}\right)\right|+\left|\psi^{\prime \prime}\left(v_{2}\right)\right|\right] .
$$


Corollary 2. If we choose $s=0$ and $\alpha=2$ in Theorem, then for $P$ function, we get

$$
\left|\frac{1}{v_{2}-v_{1}} \int_{v_{1}}^{v_{2}} \psi(\tau) d \tau-\psi\left(\frac{v_{1}+v_{2}}{2}\right)\right| \leq \frac{7\left(v_{2}-v_{1}\right)^{2}}{120}\left[\left|\psi^{\prime \prime}\left(v_{1}\right)\right|+\left|\psi^{\prime \prime}\left(v_{2}\right)\right|\right] .
$$

Remark 1. Estimates (3) and (4) show that if the function $\left|\psi^{\prime \prime}\right|$ is $s$-Godunova-Levin convex or is $P$ function, then the upper bound of the midpoint inequality is worse than the estimates available in the literature (for example, in [9] Corollary 2.1 and in [29] Proposition 1).

Lemma 3. $\psi: I=\left[v_{1}, v_{2}\right] \rightarrow \mathbb{R}$ be a differentiable function on $\left(v_{1}, v_{2}\right)$. If $\psi^{\prime} \in L\left[v_{1}, v_{2}\right]$, then $\forall \xi, \zeta \in\left[v_{1}, v_{2}\right]$, with $\xi<\zeta$ and $\alpha>0$ we have:

$$
\frac{1}{\zeta-\xi} \psi(\zeta)-\frac{\Gamma(\alpha+1)}{(\zeta-\xi)^{\alpha+1}} J_{\zeta^{-}}^{\alpha} \psi(\xi)=\int_{0}^{1}(1-\tau)^{\alpha} \psi^{\prime}(\tau \xi+(1-\tau) \zeta) d \tau
$$

Proof. By integrating parts

$$
\begin{aligned}
& \int_{0}^{1}(1-\tau)^{\alpha} \psi^{\prime}(\tau \xi+(1-\tau) \zeta) d \tau \\
& =\frac{1}{\zeta-\xi} \psi(\zeta)-\frac{\alpha}{\zeta-\xi} \int_{0}^{1}(1-\tau)^{\alpha-1} \psi(\tau \xi+(1-\tau) \zeta) d \tau
\end{aligned}
$$

and, applying the change of variable $u=\tau \xi+(1-\tau) \zeta$ to the resulting integral, we obtain

$$
\begin{aligned}
& \frac{1}{\zeta-\xi} \psi(\zeta)-\frac{\alpha}{(\zeta-\xi)^{\alpha+1}} \int_{\xi}^{\zeta}(u-\xi)^{\alpha-1} \psi(u) d u \\
& =\frac{1}{\zeta-\xi} \psi(\zeta)-\frac{\alpha}{(\zeta-\xi)^{\alpha+1}} \Gamma(\alpha) J_{\zeta^{-}}^{\alpha} \psi(\xi) .
\end{aligned}
$$

The proof is finished.

Remark 2. If we choose $\xi=v_{1}$ and $\zeta=v_{2}$ in Lemma 3, we obtain

$$
\frac{1}{v_{2}-v_{1}} \psi\left(v_{2}\right)-\frac{\alpha}{\left(v_{2}-v_{1}\right)^{\alpha+1}} \Gamma(\alpha) J_{v_{2}-}^{\alpha} \psi\left(v_{1}\right)=\int_{0}^{1}(1-\tau)^{\alpha} \psi^{\prime}\left(\tau v_{1}+(1-\tau) v_{2}\right) d \tau .
$$

Theorem 3. Let $I=\left[v_{1}, v_{2}\right] \rightarrow \mathbb{R}$, be a differentiable function on $I^{\circ}$ such that $\psi^{\prime} \in L\left[v_{1}, v_{2}\right]$, with $\xi, \zeta \in\left[v_{1}, v_{2}\right]$. If $\psi^{\prime} \in Q C([\xi, \zeta])$. Then, for all $\alpha>0$ we have

$$
\frac{1}{\zeta-\xi} \psi(\zeta)-\frac{\Gamma(\alpha+1)}{(\zeta-\xi)^{\alpha+1}} J_{\zeta^{-}}^{\alpha} \psi(\xi) \leq \frac{1}{(\alpha+1)} \max \left\{\psi^{\prime}(\xi), \psi^{\prime}(\zeta)\right\}
$$

Proof. Since $\psi^{\prime}(\tau \xi+(1-\tau) \zeta) \leq \max \left\{\psi^{\prime}(\xi), \psi^{\prime}(\zeta)\right\}$ for $\tau \in[0,1]$ and from Lemma 3, we obtain

$$
\begin{aligned}
\frac{1}{\zeta-\xi} \psi(\zeta)-\frac{\alpha}{(\zeta-\xi)^{\alpha+1}} \Gamma(\alpha) J_{\zeta^{-}}^{\alpha} \psi(\xi) & =\int_{0}^{1}(1-\tau)^{\alpha} \psi^{\prime}(\tau \xi+(1-\tau) \zeta) d \tau \\
& \leq \max \left\{\psi^{\prime}(\xi), \psi^{\prime}(\zeta)\right\} \int_{0}^{1}(1-\tau)^{\alpha} d \tau \\
& =\frac{1}{(\alpha+1)} \max \left\{\psi^{\prime}(\xi), \psi^{\prime}(\zeta)\right\}
\end{aligned}
$$

this completes the proof of theorem.

Corollary 3. Under the conditions of Theorem 3, if we choose $\xi=v_{1}$ and $\zeta=v_{2}$ and the function $\psi$ is increasing, then we get:

$$
\begin{aligned}
& \frac{1}{v_{2}-v_{1}} \psi\left(v_{2}\right)-\frac{\alpha}{\left(v_{2}-v_{1}\right)^{\alpha+1}} \Gamma(\alpha) J_{v_{2}-}^{\alpha} \psi\left(v_{1}\right) \\
\leq & \frac{1}{(\alpha+1)} \max \left\{\psi^{\prime}\left(v_{1}\right), \psi^{\prime}\left(v_{2}\right)\right\} \leq\|\psi\|_{\infty} \frac{1}{\alpha+1} .
\end{aligned}
$$


Corollary 4. In inequality (5), if we choose $\alpha=1$, we have

$$
\psi\left(v_{2}\right)-\frac{1}{v_{2}-v_{1}} \int_{v_{1}}^{v_{2}} \psi(\xi) d \xi \leq \frac{\left(v_{2}-v_{1}\right)}{2} \max \left\{\psi^{\prime}\left(v_{1}\right), \psi^{\prime}\left(v_{2}\right)\right\} .
$$

Theorem 4. Let $\psi: I=\left[v_{1}, v_{2}\right] \rightarrow \mathbb{R}$, be a differentiable function on $\left(v_{1}, v_{2}\right)$ such that $\psi^{\prime} \in L\left[v_{1}, v_{2}\right]$, $v_{1} \leq \xi<\zeta \leq v_{2}$. If $\left|\psi^{\prime}\right|^{q} \in Q C([\xi, \zeta]), q>1, p=\frac{q}{q-1}$, then $\forall \alpha>0$, we have

$$
\left|\frac{1}{\zeta-\xi} \psi(\zeta)-\frac{\alpha \Gamma(\alpha)}{(\zeta-\xi)^{\alpha+1}} J_{\zeta^{-}}^{\alpha} \psi(\xi)\right| \leq \frac{\max \left\{\left|\psi^{\prime}(\xi)\right|^{q},\left|\psi^{\prime}(\zeta)\right|^{q}\right\}^{\frac{1}{q}}}{\alpha+1} .
$$

Proof. From Lemma 3, using the properties of the modulus and Hölder's inequality, taking into account that $\left|\psi^{\prime}\right|^{q}$ is a quasiconvex function, we obtain:

$$
\begin{aligned}
& \left|\frac{1}{\zeta-\xi} \psi(\zeta)-\frac{\alpha}{(\zeta-\xi)^{\alpha+1}} \Gamma(\alpha) J_{\zeta^{-}}^{\alpha} \psi(\xi)\right|=\left|\int_{0}^{1}(1-\tau)^{\alpha} \psi^{\prime}(\tau \xi+(1-\tau) \zeta) d \tau\right| \\
& \leq \int_{0}^{1}\left|(1-\tau)^{\alpha}\right|\left|\psi^{\prime}(\tau \xi+(1-\tau) \zeta)\right| d \tau \\
& =\int_{0}^{1}(1-\tau)^{\alpha\left(1-\frac{1}{q}\right)}(1-\tau)^{\alpha \frac{1}{q}}\left|\psi^{\prime}(\tau \xi+(1-\tau) \zeta)\right| d \tau \\
& \leq\left(\int_{0}^{1}(1-\tau)^{\alpha} d \tau\right)^{\frac{1}{p}}\left(\int_{0}^{1}(1-\tau)^{\alpha}\left|\psi^{\prime}(\tau \xi+(1-\tau) \zeta)\right|^{q} d \tau\right)^{\frac{1}{q}} \\
& =\left(\frac{1}{\alpha+1}\right) \max \left\{\left|\psi^{\prime}(\xi)\right|^{q},\left|\psi^{\prime}(\zeta)\right|^{q}\right\}^{\frac{1}{q}},
\end{aligned}
$$

this completes proof.

Corollary 5. If we choose $\xi=v_{1}, \zeta=v_{2}$ and $\alpha=1$ in inequality (6), then

$$
\left|\psi\left(v_{2}\right)-\frac{1}{v_{2}-v_{1}} \int_{v_{1}}^{v_{2}} \psi(\xi) d \xi\right| \leq \frac{v_{2}-v_{1}}{2} \max \left\{\left|\psi^{\prime}\left(v_{1}\right)\right|^{q},\left|\psi^{\prime}\left(v_{2}\right)\right|^{q}\right\}^{\frac{1}{q}} .
$$

Lemma 4. Let $\psi: I=\left[v_{1}, v_{2}\right] \rightarrow \mathbb{R}$ be a differentiable function on $\left(v_{1}, v_{2}\right)$. If $\psi^{\prime} \in L\left[v_{1}, v_{2}\right]$, then for all $v_{1} \leq \xi<\zeta \leq v_{2}$ and $\alpha>0$, we have.

$$
\begin{gathered}
\frac{1}{\xi-\zeta}[\psi(\xi)-\psi(\zeta)]+\frac{\alpha \Gamma(\alpha)}{(\zeta-\xi)}\left[J_{\xi^{+}}^{\alpha} \psi(\zeta)-J_{\zeta^{-}}^{\alpha} \psi(\xi)\right] \\
=\int_{0}^{1} \tau^{\alpha} \psi^{\prime}(\tau \xi+(1-\tau) \zeta) d \tau+\int_{0}^{1}(1-\tau)^{\alpha} \psi^{\prime}(\tau \xi+(1-\tau) \zeta) d \tau
\end{gathered}
$$

Proof. Applying the method of integration by parts for each integral, we get:

$$
\begin{aligned}
& \int_{0}^{1} \tau^{\alpha} \psi^{\prime}(\tau \xi+(1-\tau) \zeta) d \tau \\
& =\frac{1}{\xi-\zeta} \psi(\xi)+\frac{\alpha}{(\zeta-\xi)^{\alpha+1}} \int_{\xi}^{\zeta}(\zeta-u)^{\alpha-1} \psi(u) d u, \quad u=\tau \xi+(1-\tau) \zeta \\
& =\frac{1}{\xi-\zeta} \psi(\xi)+\frac{\alpha \Gamma(\alpha)}{(\zeta-\xi)^{\alpha+1}} J_{\xi^{+}}^{\alpha} \psi(\zeta)
\end{aligned}
$$

and

$$
\begin{aligned}
& \int_{0}^{1}(1-\tau)^{\alpha} \psi^{\prime}(\tau \xi+(1-\tau) \zeta) d \tau \\
& =-\frac{1}{\xi-\zeta} \psi(\zeta)-\frac{\alpha}{(\zeta-\xi)^{\alpha+1}} \int_{\xi}^{\zeta}(u-\xi)^{\alpha-1} \psi(u) d u, \quad u=\tau \xi+(1-\tau) \zeta \\
& =-\frac{1}{\xi-\zeta} \psi(\zeta)-\frac{\alpha \Gamma(\alpha)}{(\zeta-\xi)^{\alpha+1}} J_{\zeta^{-}}^{\alpha} \psi(\xi) .
\end{aligned}
$$


Adding the last two equalities, we obtain (7).

Theorem 5. Let $\psi: I=\left[v_{1}, v_{2}\right] \rightarrow \mathbb{R}$ be a differentiable function on $\left(v_{1}, v_{2}\right)$. If $\psi^{\prime} \in L\left[v_{1}, v_{2}\right]$ and $\left|\psi^{\prime}\right|$ is $s-$ Godunova-Levin type function. Then, for all $v_{1} \leq \xi<\zeta \leq v_{2}$ and $\alpha>0, s \in[0,1)$ we have

$$
\begin{gathered}
\left|\frac{1}{\xi-\zeta}[\psi(\xi)-\psi(\zeta)]+\frac{\alpha \Gamma(\alpha)}{(\zeta-\xi)}\left[J_{\xi^{+}}^{\alpha} \psi(\zeta)-J_{\zeta^{-}}^{\alpha} \psi(\xi)\right]\right| \\
\leq\left[B(\alpha+1,1-s)+\frac{1}{\alpha-s+1}\right](|\psi(\xi)|+|\psi(\zeta)|),
\end{gathered}
$$

where $B(\xi, \zeta)=\int_{0}^{1} \tau^{\xi-1}(1-\tau)^{\zeta-1} d \tau, \xi>1, \zeta>0$ is Euler Beta function.

Proof. From Lemma 4 and with properties of modulus

$$
\begin{aligned}
& \left|\int_{0}^{1} \tau^{\alpha} \psi^{\prime}(\tau \xi+(1-\tau) \zeta) d \tau+\int_{0}^{1}(1-\tau)^{\alpha} \psi^{\prime}(\tau \xi+(1-\tau) \zeta) d \tau\right| \\
& \leq \int_{0}^{1} \tau^{\alpha}\left|\psi^{\prime}(\tau \xi+(1-\tau) \zeta)\right| d \tau+\int_{0}^{1}(1-\tau)^{\alpha}\left|\psi^{\prime}(\tau \xi+(1-\tau) \zeta)\right| d \tau .
\end{aligned}
$$

Since $\left|\psi^{\prime}\right|$ is $s$-Godunova-Levin type function, we get

$$
\begin{aligned}
\int_{0}^{1} \tau^{\alpha}\left|\psi^{\prime}(\tau \xi+(1-\tau) \zeta)\right| d \tau & \leq\left|\psi^{\prime}(\xi)\right| \int_{0}^{1} \tau^{\alpha-s} d \tau+\left|\psi^{\prime}(\zeta)\right| \int_{0}^{1} \tau^{\alpha}(1-\tau)^{-s} d \tau \\
& =\left[\frac{1}{\alpha-s+1}\left|\psi^{\prime}(\xi)\right|+\left|\psi^{\prime}(\zeta)\right| B(\alpha+1,1-s)\right]
\end{aligned}
$$

and

$$
\int_{0}^{1}(1-\tau)^{\alpha}\left|\psi^{\prime}(\tau \xi+(1-\tau) \zeta)\right| d \tau=\left|\psi^{\prime}(\xi)\right| B(1-s, \alpha+1)+\left|\psi^{\prime}(\zeta)\right| \frac{1}{\alpha-s+1} .
$$

Finally, since $B(\xi, \zeta)=B(\zeta, \xi)$, we have (8). This completes the proof.

Theorem 6. Let $\psi: I=\left[v_{1}, v_{2}\right] \rightarrow \mathbb{R}$, be a differentiable function on $\left(v_{1}, v_{2}\right)$ such that $\psi, g \in L\left[v_{1}, v_{2}\right]$ and $0 \leq v_{1}<v_{2}$. If $|\psi|^{p},|g|^{q} \in Q C(I)$ and increasing on $\left[v_{1}, v_{2}\right], q>1$. Then for all for $p>1, \frac{1}{p}+\frac{1}{q}=1$ the inequality

$$
\begin{aligned}
& \frac{1}{v_{2}-v_{1}}\left|\int_{v_{1}}^{v_{2}} \psi(\xi) g(\xi) h(\xi) d \xi\right| \leq \frac{\|\psi\|_{\infty}\|g\|_{\infty}}{2} \\
& \times\left[\frac{\Gamma(\alpha+1)}{\left(v_{2}-v_{1}\right)^{\alpha+1}}\left[J_{v_{1}^{+}}^{\alpha} \psi\left(v_{2}\right)+J_{v_{2}^{-}}^{\alpha} \psi\left(v_{1}\right)\right]-\frac{1}{v_{2}-v_{1}} \int_{v_{1}}^{v_{2}} \psi(\xi) d \xi\right]
\end{aligned}
$$

holds, where

$$
h\left(\tau v_{1}+(1-\tau) v_{2}\right)=\left[(1-\tau)^{\alpha}+\left(\tau^{\alpha}-1\right)\right] \psi\left(\tau v_{1}+(1-\tau) v_{2}\right) \geq 0
$$

$\forall \tau \in[0,1]$ and $\alpha \in[0,1]$.

Proof. The following equality is obvious:

$$
\begin{aligned}
& \frac{1}{v_{2}-v_{1}} \int_{v_{1}}^{v_{2}} \psi(\xi) g(\xi) h(\xi) d \xi \\
= & \int_{0}^{1} \psi\left(\tau v_{1}+(1-\tau) v_{2}\right) g\left(\tau v_{1}+(1-\tau) v_{2}\right) h\left(\tau v_{1}+(1-\tau) v_{2}\right) d \tau
\end{aligned}
$$


Now, we use the weighted Hölder inequality:

$$
\begin{aligned}
& \frac{1}{v_{2}-v_{1}}\left|\int_{v_{1}}^{v_{2}} \psi(\xi) g(\xi) h(\xi) d \xi\right| \leq\left(\int_{0}^{1}\left|\psi\left(\tau v_{1}+(1-\tau) v_{2}\right)\right|^{p} h\left(\tau v_{1}+(1-\tau) v_{2}\right) d \tau\right)^{\frac{1}{p}} \\
& \times\left(\int_{0}^{1}\left|g\left(\tau v_{1}+(1-\tau) v_{2}\right)\right|^{q} h\left(\tau v_{1}+(1-\tau) v_{2}\right) d \tau\right)^{\frac{1}{q}} \\
& \leq\left[\max \left\{\left|\psi\left(v_{1}\right)\right|^{p},\left|\psi\left(v_{2}\right)\right|^{p}\right\}\right]^{\frac{1}{p}}\left[\max \left\{\left|g\left(v_{1}\right)\right|^{q},\left|g\left(v_{2}\right)\right|^{q}\right\}\right]^{\frac{1}{q}} \\
& \times\left(\int_{0}^{1} h\left(\tau v_{1}+(1-\tau) v_{2}\right) d \tau\right)^{\frac{1}{p}}\left(\int_{0}^{1} h\left(\tau v_{1}+(1-\tau) v_{2}\right) d \tau\right)^{\frac{1}{q}} \\
& =\left[\max \left\{\left|\psi\left(v_{1}\right)\right|^{p},\left|\psi\left(v_{2}\right)\right|^{p}\right\}\right]^{\frac{1}{p}}\left[\max \left\{\left|g\left(v_{1}\right)\right|^{q},\left|g\left(v_{2}\right)\right|^{q}\right\}\right]^{\frac{1}{q}} \\
& \times\left(\int_{0}^{1} h\left(\tau v_{1}+(1-\tau) v_{2}\right) d \tau\right)^{\frac{1}{p}+\frac{1}{q}} \\
& =\left[\max \left\{\left|\psi\left(v_{1}\right)\right|^{p},\left|\psi\left(v_{2}\right)\right|^{p}\right\}\right]^{\frac{1}{p}}\left[\max \left\{\left|g\left(v_{1}\right)\right|^{q},\left|g\left(v_{2}\right)\right|^{q}\right\}\right]^{\frac{1}{q}} \\
& \times\left(\int_{0}^{1} h\left(\tau v_{1}+(1-\tau) v_{2}\right) d \tau\right) \\
& =\|\psi\|_{\infty}\|g\|_{\infty}\left(\int_{0}^{1} h\left(\tau v_{1}+(1-\tau) v_{2}\right) d \tau\right) \\
& =\|\psi\|_{\infty}\|g\|_{\infty}\left(\int_{0}^{1}\left[(1-\tau)^{\alpha}+\left(\tau^{\alpha}-1\right)\right] \psi\left(\tau v_{1}+(1-\tau) v_{2}\right) d \tau\right) \\
& =\frac{\|\psi\|_{\infty}\|g\|_{\infty}}{2}\left[\frac{\Gamma(\alpha+1)}{\left(v_{2}-v_{1}\right)^{\alpha+1}}\left[J_{v_{2}^{-}}^{\alpha} \psi\left(v_{1}\right)+J_{v_{1}^{+}}^{\alpha} \psi\left(v_{2}\right)\right]-\frac{1}{v_{2}-v_{1}} \int_{v_{1}}^{v_{2}} \psi(\xi) d \xi\right] \text {, }
\end{aligned}
$$

this completes the proof.

Theorem 7. Let $\psi: I=\left[v_{1}, v_{2}\right] \rightarrow \mathbb{R}$, be a differentiable function on $\left(v_{1}, v_{2}\right)$. If $\left|\psi^{\prime}\right|^{q} \in Q C(I)$ and $\left|\psi^{\prime}\right|^{q}$ is increasing. Then for all $q>1, \frac{1}{p}+\frac{1}{q}=1, \alpha \in[0,1]$ the inequality

$$
\begin{gathered}
\Gamma(\alpha+1)\left(J_{v_{1}^{+}}^{\alpha} \psi\left(v_{2}\right), J_{v_{2}^{-}}^{\alpha} \psi\left(v_{1}\right)\right)_{\alpha \in[0,1]} \\
\leq \frac{v_{2}-v_{1}}{2}\left(\frac{1}{\alpha p^{2}-\alpha p+\alpha+1}\right)^{\frac{1}{p}}\left(\frac{1}{\alpha-\alpha p+1}\right)^{\frac{1}{q}}\left\|\psi^{\prime}\right\|_{\infty}
\end{gathered}
$$

holds, where

$$
\begin{aligned}
& \Gamma(\alpha+1)\left(J_{v_{1}^{+}}^{\alpha} \psi\left(v_{2}\right), J_{v_{2}^{-}}^{\alpha} \psi\left(v_{1}\right)\right)_{\alpha \in[0,1]} \\
= & \left|\frac{\psi\left(v_{1}\right)+\psi\left(v_{2}\right)}{2}-\frac{\Gamma(\alpha+1)}{2\left(v_{2}-v_{1}\right)^{\alpha}}\left[J_{v_{1}^{+}}^{\alpha} \psi\left(v_{2}\right)+J_{v_{2}^{-}}^{\alpha} \psi\left(v_{1}\right)\right]\right| .
\end{aligned}
$$

Proof. From (2), taking into account the properties of the module, we get:

$$
\Gamma(\alpha+1)\left(J_{v_{1}^{+}}^{\alpha} \psi\left(v_{2}\right), J_{v_{2}^{-}}^{\alpha} \psi\left(v_{1}\right)\right)_{\alpha \in[0,1]}
$$




$$
\leq \frac{v_{2}-v_{1}}{2}\left[\int_{0}^{1}\left|(1-\tau)^{\alpha}-\tau^{\alpha}\right|\left|\psi^{\prime}\left(\tau v_{1}+(1-\tau) v_{2}\right)\right| d \tau\right] .
$$

We know that for $\alpha \in[0,1]$ and $\forall \tau_{1}, \tau_{2} \in[0,1]$,

$$
\left|\tau_{1}^{\alpha}-\tau_{2}^{\alpha}\right| \leq\left|\tau_{1}-\tau_{2}\right|^{\alpha},
$$

that is

$$
\int_{0}^{1}\left|\left(1-\tau^{\alpha}\right)-\tau^{\alpha}\right| d \tau \leq \int_{0}^{1}|1-2 \tau|^{\alpha} d \tau .
$$

Taking into account last inequality and using the power mean inequality for (10), we obtain:

$$
\begin{aligned}
& \Gamma(\alpha+1)\left(J_{v_{1}^{+}}^{\alpha} \psi\left(v_{2}\right), J_{v_{2}^{-}}^{\alpha} \psi\left(v_{1}\right)\right)_{\alpha \in[0,1]} \\
& \leq \frac{v_{2}-v_{1}}{2}\left[\int_{0}^{1}|1-2 \tau|^{\alpha}\left|\psi^{\prime}\left(\tau v_{1}+(1-\tau) v_{2}\right)\right| d \tau\right] \\
& =\frac{v_{2}-v_{1}}{2}\left[\int_{0}^{1}|1-2 \tau|^{\alpha p}\left|\psi^{\prime}\left(\tau v_{1}+(1-\tau) v_{2}\right)\right||1-2 \tau|^{\alpha(1-p)} d \tau\right] \\
& \leq \frac{v_{2}-v_{1}}{2}\left(\int_{0}^{1}|1-2 \tau|^{\alpha p^{2}}|1-2 \tau|^{\alpha(1-p)} d \tau\right)^{\frac{1}{p}} \\
& \times\left(\int_{0}^{1}\left|\psi^{\prime}\left(\tau v_{1}+(1-\tau) v_{2}\right)\right|^{q}|1-2 \tau|^{\alpha(1-p)} d \tau\right)^{\frac{1}{q}} \\
& =\frac{v_{2}-v_{1}}{2}\left(\int_{0}^{1}|1-2 \tau|^{\alpha p^{2}+\alpha(1-p)} d \tau\right)^{\frac{1}{p}}\left(\int_{0}^{1}|1-2 \tau|^{\alpha(1-p)} d \tau\right)^{\frac{1}{q}} \\
& \times\left(\max \left\{\left|\psi^{\prime}\left(v_{1}\right)\right|^{q},\left|\psi^{\prime}\left(v_{2}\right)\right|^{q}\right\}\right)^{\frac{1}{q}} .
\end{aligned}
$$

Since

$$
\begin{aligned}
\int_{0}^{1}|1-2 \tau|^{\alpha\left(p^{2}-p-1\right)} d \tau & =\int_{0}^{\frac{1}{2}}(1-2 \tau)^{\alpha\left(p^{2}-p-1\right)} d \tau+\int_{\frac{1}{2}}^{1}(2 \tau-1)^{\alpha\left(p^{2}-p-1\right)} d \tau \\
& =\frac{1}{\alpha p^{2}-\alpha p+\alpha+1}, \\
\int_{0}^{1}|1-2 \tau|^{\alpha(1-p)} d \tau & =\int_{0}^{\frac{1}{2}}(1-2 \tau)^{\alpha(1-p)} d \tau+\int_{\frac{1}{2}}^{1}(2 \tau-1)^{\alpha(1-p)} d \tau \\
& =\frac{1}{\alpha-\alpha p+1} .
\end{aligned}
$$

Finally, we get

$$
\begin{aligned}
& \Gamma(\alpha+1)\left(J_{v_{1}^{+}}^{\alpha} \psi\left(v_{2}\right), J_{v_{2}^{-}}^{\alpha} \psi\left(v_{1}\right)\right)_{\alpha \in[0,1]} \\
& \leq \frac{v_{2}-v_{1}}{2}\left(\frac{1}{\alpha p^{2}-\alpha p+\alpha+1}\right)^{\frac{1}{p}}\left(\frac{1}{\alpha-\alpha p+1}\right)^{\frac{1}{q}} \\
& \times\left(\max \left\{\left|\psi^{\prime}\left(v_{1}\right)\right|^{q},\left|\psi^{\prime}\left(v_{2}\right)\right|^{q}\right\}\right)^{\frac{1}{q}} \\
& \leq \frac{v_{2}-v_{1}}{2}\left(\frac{1}{\alpha p^{2}-\alpha p+\alpha+1}\right)^{\frac{1}{p}}\left(\frac{1}{\alpha-\alpha p+1}\right)^{\frac{1}{q}}\left\|\psi^{\prime}\right\|_{\infty},
\end{aligned}
$$

this completes the required proof. 
Remark 3. For $p \in(1, \infty)$, we have

$$
\lim _{p \rightarrow \infty}\left(\frac{1}{1+\alpha-\alpha p}\right)^{1-\frac{1}{p}}=1, \quad \lim _{p \rightarrow 1^{+}}\left(\frac{1}{1+\alpha-\alpha p}\right)^{\frac{1}{q}}=1 .
$$

and

$$
\begin{aligned}
\lim _{p \rightarrow \infty}\left(\frac{1}{\alpha p^{2}-\alpha p+\alpha+1}\right)^{\frac{1}{p}}=1, \lim _{p \rightarrow 1^{+}}\left(\frac{1}{\alpha p^{2}-\alpha p+\alpha+1}\right)^{\frac{1}{p}}=\frac{1}{\alpha+1}<1, \\
\frac{1}{\alpha+1}<\left(\frac{1}{\alpha p^{2}-\alpha p+\alpha+1}\right)^{\frac{1}{p}}<1,
\end{aligned}
$$

Thus, we can rewrite inequality (9) as follows

$$
\left|\frac{\psi\left(v_{1}\right)+\psi\left(v_{2}\right)}{2}-\frac{\Gamma(\alpha+1)}{2\left(v_{2}-v_{1}\right)^{\alpha}}\left[J_{v_{1}^{+}}^{\alpha} \psi\left(v_{2}\right)+J_{v_{2}^{-}}^{\alpha} \psi\left(v_{1}\right)\right]\right| \leq \frac{v_{2}-v_{1}}{2}\left\|\psi^{\prime}\right\|_{\infty} .
$$

Applications to special means

In this section, we consider some special means for arbitrary real numbers:

1 Arithmetic mean:

$$
A\left(v_{1}, v_{2}\right)=\frac{v_{1}+v_{2}}{2}, \quad v_{1}, v_{2} \in \mathbb{R}^{+}
$$

2 Logarithmic mean:

$$
L\left(v_{1}, v_{2}\right)=\frac{v_{1}-v_{2}}{\ln \left|v_{1}\right|-\ln \left|v_{2}\right|}, \quad v_{1} \neq v_{2}, v_{1}, v_{2} \neq 0, v_{1}, v_{2} \in \mathbb{R}^{+},
$$

3 Generalized log-mean:

$$
L_{n}\left(v_{1}, v_{2}\right)=\left[\frac{v_{2}^{n+1}-v_{1}^{n+1}}{(n+1)\left(v_{2}-v_{1}\right)}\right]^{\frac{1}{n}}, \quad n \in \mathbb{Z} \backslash\{-1,0\}, v_{1}, v_{2} \in \mathbb{R}^{+} .
$$

Now, using the some results, we give some applications to special means of real numbers.

Proposition 1. Let $v_{1}, v_{2} \in \mathbb{R}^{+}, v_{1}<v_{2}$ and $n \in \mathbb{Z}$. Then, we have

$$
v_{2}^{n}-L_{n}^{n}\left(v_{1}, v_{2}\right) \leq 0.5 n\left(v_{2}-v_{1}\right) \max \left\{v_{1}^{n-1}, v_{2}{ }^{n-1}\right\} .
$$

Proof. The proof follows from the Corollary 4 applied to the map $f(x)=x^{n}, x \in \mathbb{R}$.

Proposition 2. Let $v_{1}, v_{2} \in \mathbb{R}^{+}, v_{1}<v_{2}$ and $n \in \mathbb{Z}$. Then, for all $q \geq 1$, we have

$$
\left|v_{2}^{n}-L_{n}^{n}\left(v_{1}, v_{2}\right)\right| \leq 0.5 n\left(v_{2}-v_{1}\right)\left(\max \left\{\left(\left|v_{1}\right|^{n-1}\right)^{q},\left(\left|v_{2}\right|^{n-1}\right)^{q}\right\}\right)^{\frac{1}{q}} .
$$

Proof. The assertion follows from Corollary 5 applied to the quasi-convex mapping $\psi(\xi)=\xi^{n}, \xi \in \mathbb{R}$.

\section{References}

1 Годунова Е.К. Неравенства для функций широкого класса, содержащего выпуклые, монотонные и некоторые другие виды функций / Е.К. Годунова, В.И. Левин // Вычисл. мат. и мат. физ.: межвуз. сб. науч. тр. - М.: МГПИ, 1985. - С. 138-142.

2 Dragomir S.S. Some Inequalities of Hadamard Type / S.S. Dragomir, J. Pecariĉ, L. Persson // Soochow J. Math. - 1995. - 21(3). - P. 335-341.

3 Dragomir S.S. Selected Topics on Hermite-Hadamard Inequalities and Applications / S.S. Dragomir, C.E.M. Pearse // RGMIA Monographs, Victoria University. - 2000.

Retrieved from http://rgmia.org/papers/monographs/Master.pdf. 
4 Butt S.I. Several New Integral Inequalities Via $k$-Riemann-Liouville Fractional Integrals Operators / S.I. Butt, B. Bayraktar, M. Umar // Probl. Anal. Issues Anal. - 2021. - 10 (28). — No. 1. - P. 3-22.

5 Khan T.U. Generalized conformable fractional operators / T.U. Khan, M.A. Khan // J. Comput. Appl. Math. - 2019. - 346. - P. 378-389.

6 Napoles J.E. New Hermite-Hadamard Type Inequalities Involving Non-Conformable Integral Operators / J.E. Napoles Valdes, J.M. Rodriguez, J.M. Sigarreta // Symmetry 11(9). - 2019. - 1108.

7 Farid G. Some Generalized k-Fractional Integral Inequalities for Quasi-Convex Functions / G. Farid, C. Yong Jung, S. Ullah, W. Nazeer, M. Waseem, S. Min Kang // J. Computational Analysis And applications. - 2021. - Vol. 29. - No. 3. - P. 454-467.

8 Set E. Certain new Hermite-Hadamard type inequalities for convex functions via fractional integrals / E. Set, M.E. Özdemir, N. Korkut // Commun. Fac. Sci. Univ. Ank. Ser. A1 Math. Stat. - 2019. Vol. 68. - No. 1. - P. 61-69.

9 Bayraktar B. Some Integral Inequalities Of Hermite-Hadamard Type For Differentiable $(s, m)-$ Convex Functions Via Fractional Integrals / B. Bayraktar // TWMS J. App. Eng. Math. - 2020. — 10(3). P. 625-637.

10 Bayraktar B. Some New Generalizations Of Hadamard-Type Midpoint Inequalities Involving Fractional Integrals / B. Bayraktar // Probl. Anal. Issues Anal. - 2020. - 9 (27-3). - P. 66-82.

11 Bayraktar B. Some New Inequalities of Hermite-Hadamard Type for Differentiable Godunova-Levin Functions via Fractional Integrals / B. Bayraktar // Konuralp Journal of Mathematics. — 2020. — 8(1). - P. 91-96.

12 Bayraktar B. Generalization Of Hadamard-Type Trapezoid Inequalities For Fractional Integral Operators / B. Bayraktar, M.E. Özdemir // Ufa Mathematical Journal. - 2021. - 13(1). — 119-13011.

13 Belarbi S. On some new fractional integral inequalities / S. Belarbi, Z. Dahmani // J. Ineq. Pure and Appl. Math. - 2009. - Vol. 10. - No. 3. - Art. 86.

14 Dahmani Z. New generalizations of Gruss inequality using Riemann-Liouville fractional integrals / Z. Dahmani, L. Tabharit, S. Taf // Bull. Math. Anal. Appl. - 2010. - Vol. 2. - No. 3. - P. 93-99.

15 Sarıkaya M. Z. Hermite-Hadamard's inequalities for fractional integrals and related fractional inequalities / M.Z. Sarıkaya, E. Set, H. Yaldız, N. Başak // Math. and Comput. Mod. - 2013. - Vol. 57. - No. 9-10. - P. 2403-2407.

16 Ullah S. Generalized fractional inequalities for quasi-convex functions / S. Ullah, G. Farid, K. A. Khan, A. Waheed, S. Mehmood // Adv. Difference Equ. - 2019. - Paper No. 15. - 16 pages.

17 Dragomir S.S. Quasi-convex functions and Hadamard's inequality / S.S. Dragomir, C.E.M. Pearce // Bull. Austral. Math. Soc. - 1998. - 57. - P. 377-385.

18 Ion D.A. Some estimates on the Hermite-Hadamard inequality through quasi-convex functions / D.A. Ion // Annals of University of Craiova, Math. Sci. Ser. - 2007. - 34. - P. 82-87.

19 Hussain S. New Integral Inequalities of the Type of Hermite-Hadamard Through Quasi Convexity / S. Hussain, S. Qaisar // Punjab University Journal of Mathematics. - 2013. - Vol. 45. - P. 33-38.

20 Nwaeze, Eze R. Integral inequalities via generalized quasiconvexity with applications / Eze R. Nwaeze // Journal of Inequalities and Applications. - 2019. - Vol. 2019. - No. 236.

21 Latif M.A. New Inequalities of Fejer' and Hermite-Hadamard type Concerning Convex and Quasi-Convex Functions With Applications / M.A. Latif, S.S. Dragomir, S. Obeidat // Punjab University Journal of Mathematics. - 2021. - Vol. 53(2). - P. 1-17.

22 Bayraktar B. Some Generalized Hadamard Type Inequalities Via Fractional Integrals / B. Bayraktar, A. Attaev, V. Kudaev // Russian Mathematics (Iz. VUZ). - 2021. - 65(2). - P. 1-14.

23 Noor M.D. Hermite-Hadamard Inequalities For s-Godunova-Levin Preinvex Functions / M.D. Noor, K.A. Noor, M.U. Awan, S. Khan // J. Adv. Math. Stud. - 2014. - 7(2). - P. 12-19.

24 Noor M.A. Fractional Hermite-Hadamard Inequalities for some New Classes of Godunova-Levin Functions / M.A. Noor, K.I. Noor, M.U. Awan, S. Khan // Appl. Math. Inf. Sci. - 2014. - 8(6). - P. 2865-2872.

25 Özdemir M.E. Some inequalities for the $s$-Godunova-Levin type functions / M.E. Özdemir // Math Sci. -2015 . - 9. - P. 27-32. 
26 Li M. Some Fractional Hermite-Hadamard Inequalities for Convex and Godunova-Levin Functions / M. Li, J. Wang, W. Wei // Facta Universitatis (NIŜ) Ser. Math. Inform. - 2015. - 30(2). — P. $195-208$.

27 Awan M.U. Fractional Hermite-Hadamard Inequalities for Differentiable $s$-Godunova-Levin Functions / M.U. Awan, M.A. Noor, M.V. Mihai, K.I. Noor // Filomat. - 2016. - 30(12). - P. 3235-3241.

28 Dragomir S.S. Inequalities for Beta and Gamma functions via some classical and new integral inequalities / S.S. Dragomir, R.P. Agarwal, N.S. Barnet // J. of Inequalities \& Appl. — 2000. - Vol. 5. - P. $103-165$.

29 Sarıkaya M.Z. On the generalization of some integral inequalities and their applications / M.Z. Sarıkaya, N. Aktan // Math. Comput. Modelling. - 2011. - 54. - P. 2175-2182.

30 Bayraktar B. Some New Integral Inequalities For $(s, m)$-Convex And $(\alpha, m)$-Convex Functions / B. Bayraktar, V. Kudaev // Bulletin of the Karaganda Unıversity - Mathematics. - 2019. - 94(2). - P. 15-25.

31 Özdemir M.E. Several integral inequalities for $(\alpha, s, m)$-convex functions / M.E. Özdemir, S.I. Butt, B. Bayraktar, J. Nasir // AIMS Mathematics. - 2020. - 5(4). - P. 3906-3921.

32 Jarad F. On a new class of fractional operators / F. Jarad, E. Ugurlu, T. Abdeljawad, D. Baleanu // Adv. Difference Equ. - 2017. - 2017. - Paper No. 247.

\author{
Б.Р. Байрактаров ${ }^{1}$, А.Х. Аттаев ${ }^{2}$ \\ ${ }^{1}$ Улудаг университеті, Бурса, Түркия; \\ ${ }^{2}$ РҒА КБҒО ҚМАИ, Налъчик, Ресей
}

\title{
Кейбір дөңес функциялар үшін бөлшекті-интегралды теңсіздіктер
}

\begin{abstract}
Мақалада екінші мағынадағы дөңес s - Годунов-Левин функциялары үшін және квази-дөңес функциялар үшін Риман-Лиувиллдің бөлшекті интегралдары арқылы бірнеше жаңа интегралдық теңсіздіктер алынған. Нәтижелер классикалық Гельдер теңсіздігін, Эрмит-Адамардың екі еселі теңсіздігін, орташа дәрежелік және өлшенген Гельдер теңсіздігін арқылы алынды. Соның ішінде кейбір арнайы есептеу құралдарына арналған нәтижелердің қолданулары берілген. Мақалада кезкелген нақты сандар үшін арнайы жағдайлардың қолданылулары берілген: арифметикалық, логарифмдік, жалпыланған логарифмдік жағдай.
\end{abstract}

Kiлm сөздер: интегралдық теңсіздік, Эрмит-Адамар теңсіздігі, дөңес функция, квази-дөңес функция, бөлшекті интеграл, Гельдер теңсіздігі, орташа дәрежелік теңсіздік.

\author{
Б.Р. Байрактаров ${ }^{1}$, А.Х. Аттаев² \\ ${ }^{1}$ Университет Улудаг, Бурса, Туриия; \\ ${ }^{2}$ ИПМА КБНЦ РАН, Налъчик, Россия
}

\section{Дробно-интегральные неравенства для некоторых выпуклых функций}

\begin{abstract}
В статье получены несколько новых интегральных неравенств с помощью дробных интегралов Римана-Лиувилля для выпуклых s-Годунова-Левина функций во втором смысле и для квазивыпуклых функций. Результаты получены с использованием двойного неравенства Эрмита-Адамара, классических неравенств Гёльдера, среднего степенного и взвешенного неравенства Гёльдера. В том числе дано приложение результатов для некоторых специальных вычислительных средств. Авторами приведены приложения некоторых специальных случаев для произвольных действительных чисел: арифметический случай, логарифмический случай, случай обобщённого логарифма.
\end{abstract}

Ключевые слова: интегральное неравенство, неравенство Эрмита-Адамара, выпуклая функция, квазивыпуклая функция, дробный интеграл, неравенство Гёльдера, среднее степенное неравенство. 


\section{References}

1 Godunova, E.K., \& Levin, V.I. (1985). Neravenstva dlia funktsii shirokogo klassa, soderzhashchego vypuklye, monotonnye i nekotorye drugie vidy funktsii [Inequalities for functions of a broad class that contains convex, monotone and some other forms of functions]. Vychislitelnaia matematika $i$ matematichskaia fizika: mezhvuzovskii sbornik nauchnykh trudov - Numerical mathematics and mathematical physics. Moscow: Moskovskii Gosudarstvennyi Pedagogicheskii Institut, 166, 138-142 [in Russian].

2 Dragomir, S.S., Pecariĉ, J. \& Persson, L. (1995). Some Inequalities of Hadamard Type. Soochow J. Math. $21(3), 335-341$.

3 Dragomir, S.S. \& Pearse, C.E.M., (2000). Selected Topics on Hermite-Hadamard Inequalities and Applications. RGMIA Monographs, Victoria University. rgmia.org.

Retrieved from http://rgmia.org/papers/monographs/Master.pdf.

4 Butt, S.I. Bayraktar, B. \& Umar, M. (2021). Several New Integral Inequalities Via $k$-Riemann-Liouville Fractional Integrals Operators. Probl. Anal. Issues Anal. 10(28), No. 1, 3-22.

5 Khan, T.U. \& Khan, M.A. (2019). Generalized conformable fractional operators. J. Comput. Appl. Math. 346, 378-389.

6 Napoles Valdes, J.E., Rodriguez, J.M., \& Sigarreta, J.M. (2019). New Hermite-Hadamard Type Inequalities Involving Non-Conformable Integral Operators. Symmetry 11(9), 1108.

7 Farid, G., Yong Jung, C., Ullah, S., Nazeer, W., Waseem, M., \& Min Kang, S. (2021). Some Generalized kFractional Integral Inequalities for Quasi-Convex Functions. J. Computational Analysıs and Applications., 29, 3, 454-467.

8 Set, E., Özdemir, M.E., \& Korkut, N. (2019). Certain new Hermite-Hadamard type inequalities for convex functions via fractional integrals. Commun. Fac. Sci. Univ. Ank. Ser. A1 Math. Stat., 68, 1, 61-69.

9 Bayraktar, B. (2020). Some Integral Inequalities Of Hermite-Hadamard Type For Differentiable $(s, m)-$ Convex Functions Via Fractional Integrals. TWMS J. App. Eng. Math. 10(3), 625-637.

10 Bayraktar, B. (2020). Some New Generalizations Of Hadamard-Type Midpoint Inequalities Involving Fractional Integrals. Probl. Anal. Issues Anal. 9(27-3), 66-82.

11 Bayraktar, B. (2020). Some New Inequalities of Hermite-Hadamard Type for Differentiable GodunovaLevin Functions via Fractional Integrals. Konuralp Journal of Mathematics. 8(1), 91-96.

12 Bayraktar, B., \& Özdemir, M.E. (2021). Generalization Of Hadamard-Type Trapezoid Inequalities For Fractional Integral Operators. Ufa Mathematical Journal, 13(1), 119-13011.

13 Belarbi, S., \& Dahmani, Z. (2009). On some new fractional integral inequalities. J. Ineq. Pure and Appl. Math. 10, 3, Art. 86.

14 Dahmani, Z., Tabharit, L., \& Taf, S. (2010). New generalizations of Gruss inequality using RiemannLiouville fractional integrals. Bull. Math. Anal. Appl. 2, 3, 93-99.

15 Sarıkaya, M.Z., Set, E., Yaldız, H., \& Başak, N. (2013). Hermite-Hadamard's inequalities for fractional integrals and related fractional inequalities. Math. and Comput. Mod. 57, 9-10, 2403-2407.

16 Ullah, S., Farid, G., Khan, K.A., Waheed, A., \& Mehmood, S. (2019). Generalized fractional inequalities for quasi-convex functions. Adv. Difference Equ. Paper, 15,

17 Dragomir, S.S., \& Pearce, C.E.M. (1998). Quasi-convex functions and Hadamard's inequality. Bull. Austral. Math. Soc. 57, 377-385.

18 Ion, D.A. (2007). Some estimates on the Hermite-Hadamard inequality through quasi-convex functions. Annals of University of Craiova, Math. Sci. Ser. 34, 82-87.

19 Hussain, S., \& Qaisar, S. (2013). New Integral Inequalities of the Type of Hermite-Hadamard Through Quasi Convexity. Punjab University Journal of Mathematics, 45, 33-38.

20 Nwaeze, Eze R. (2019). Integral inequalities via generalized quasiconvexity with applications. Journal of Inequalities and Applications, 2019, 236.

21 Latif, M.A., Dragomir, S.S. \& Obeidat, S. (2021). New Inequalities of Fejer' and Hermite-Hadamard type Concerning Convex and Quasi-Convex Functions With Applications. Punjab University Journal of Mathematics, 53(2), 1-17.

22 Bayraktar, B., Attaev, A., \& Kudaev, V. (2021). Some Generalized Hadamard Type Inequalities Via Fractional İntegrals. Russian Mathematics (Iz. VUZ). 65(2), 1-14. 
23 Noor, M.D., Noor, K.A., Awan, M.U., \& Khan, S. (2014). Hermite-Hadamard Inequalities For s-Godunova-Levin Preinvex Functions. J. Adv. Math. Stud. 7(2), 12-19.

24 Noor, M.A., Noor, K.I., Awan, M.U., \& Khan, S. (2014). Fractional Hermite-Hadamard Inequalities for some New Classes of Godunova-Levin Functions. Appl. Math. Inf. Sci. 8(6), 2865-2872.

25 Özdemir, M.E. (2015). Some inequalities for the s-Godunova-Levin type functions. Math Sci. 9, 27-32.

26 Li, M., Wang, J., \& Wei, W. (2015). Some Fractional Hermite-Hadamard Inequalities for Convex and Godunova-Levin Functions. Facta Universitatis (NIŜ) Ser. Math. Inform. 30(2), 195-208.

27 Awan, M.U., Noor, M.A., Mihai, M.V., \& Noor, K.I. (2016). Fractional Hermite-Hadamard Inequalities for Differentiable $s$-Godunova-Levin Functions. Filomat. 30(12), 3235-3241.

28 Dragomir, S.S., Agarwal, R.P., \& Barnet, N.S. (2000). Inequalities for Beta and Gamma functions via some classical and new integral inequalities. J. of Inequalities $\&$ Appl, 5, 103-165.

29 Sarıkaya, M.Z., \& Aktan, N. (2011). On the generalization of some integral inequalities and their applications. Math. Comput. Modelling. 54, 2175-2182.

30 Bayraktar, B., \& Kudaev, V. (2019). Some New Integral Inequalities For $(s, m)$-Convex And $(\alpha, m)$ Convex Functions. Bulletin of the Karaganda Unvversity - Mathematics. 94(2), 15-25.

31 Özdemir, M.E., Butt, S.I., Bayraktar, B., \& Nasir, J. (2020). Several integral inequalities for $(\alpha, s, m)$ convex functions. AIMS Mathematics. 5(4), 3906-3921.

32 Jarad, F., Ugurlu, E., Abdeljawad, T., \& Baleanu, D. (2017). On a new class of fractional operators. Adv. Difference Equ. 2017, Paper No. $24 \%$. 